\title{
Reception of Modulated Neutrino Day-Night Effect Signal by Neutrino Detectors
}

\author{
Oleg Kharlanov ${ }^{1, *}$ \\ ${ }^{1}$ Lomonosov Moscow State University, Faculty of Physics, 1/2 Leninskie Gory, 119991 Moscow, \\ Russia
}

\begin{abstract}
The regeneration effect of solar neutrinos in the Earth leading to the so-called day-night effect strongly depends on the neutrino energy, the time of day, and the season. Classical neutrino experiments, such as SuperKamiokande, typically observe this effect cumulatively, i.e., virtually integrate it over the year. We discuss various day-night effects that could become potentially observable if time-weighted data processing is applied to neutrino events. The procedure is similar to reception of radio-frequency modulated signals and 'demodulation' of the neutrino signal, for example, is able to reveal interesting signatures in the high-energy tail of the electron recoil energy spectrum
\end{abstract}

\section{Introduction}

In a superheterodyne receiver, an amplitude-modulated signal $f(t)=A(t) \cos \omega_{0} t$, being a product of an audible-frequency signal $A(t)$ and a high-frequency carrier wave, is usually received via a nonlinear process called mixing. In this process, the signal $f(t)$ coming from the antenna is contracted (multiplied and integrated over time) with another carrier wave $\tilde{A} \cos \omega_{0} t$ generated by the receiver itself, the result giving the sound $A(t)$ playing in the speakers. A Morse code receiver, in contrast, is only interested in the presence of the carrier wave, i.e., whether $A(t)=0$ or $A(t) \neq 0$, so it does not need the mixer circuit; however, such a 'naïve' processing of the input signal loses a lot of information carried by it.

Observation ('reception') of the regeneration effect of solar neutrinos inside the Earth has similar mathematical features. Namely, even though neutrino absorption inside it is virtually zero, propagation inside the Earth modifies the neutrino oscillation patterns in a way that the flavor composition on the exit from the Earth becomes a function of the neutrino path inside of it. This path depends on the position of the Sun, i.e., on the time of night and the season; moreover, on the time scale of typical neutrino experiments, i.e., years, the regeneration signal can be considered a rapidly-oscillating function (a 'radio-frequency' signal). The socalled day-night effect (or day-night asymmetry) $[1,2]$ observed by the Super-Kamiokande collaboration in 2014 [3] results from a simple time averaging of all the neutrinos observed during the night and further subtraction of daytime neutrinos. The question we are studying in the present paper is whether one can extract more information from the input neutrino signal by 'tuning in' to its 'carrier wave'.

\footnotetext{
*e-mail: kharlanov@physics.msu.ru
} 


\section{Temporally localized contributions to the day-night asymmetry}

We will work within the two-flavor approximation and use the spherically-symmetric PREM model of the Earth [4], for which the matrix ODE on the flavor evolution matrix $R\left(x, x_{0} ; E\right)$

$$
\mathrm{i} \lambda \partial_{x} R\left(x, x_{0}\right)=\left\{\left(-\cos 2 \theta_{0}+\frac{2 E V(x)}{\Delta m^{2}}\right) \sigma_{1}+\sin 2 \theta_{0} \sigma_{3}\right\} R\left(x, x_{0}\right), \quad R\left(x_{0}, x_{0}\right)=\mathbb{1}
$$

contains a piecewise-smooth Wolfenstein potential $V(x)=G_{\mathrm{F}} \sqrt{2} n_{e}\left(r\left(x, \Theta_{\mathrm{N}}\right)\right)$ [5] for each particular neutrino trajectory, the distance $r$ from the center of the Earth depending on the coordinate $x$ along the ray and the nadir angle $\Theta_{\mathrm{N}}(t)$. The ${ }^{8} \mathrm{~B}$ neutrinos we will study here have energies $E=5-15 \mathrm{MeV}$ corresponding to vacuum oscillation lengths $\ell_{\mathrm{osc}}=4 \pi E / \Delta m^{2} \equiv$ $\pi / \lambda$ of several hundred kilometers (the latter are also approximately equal to the oscillation lengths inside the Earth). The neutrino mixing parameters above $\operatorname{are~}^{2} \sin ^{2} 2 \theta_{0} \approx 0.86$ and $\Delta m^{2} \approx 7.6 \times 10^{-5} \mathrm{eV}^{2}$. In a number of papers (see, e.g., [6, 7]), the above equation was solved leading to the following $v_{e}$ observation probabilities

$$
\begin{gathered}
P_{\text {day }}(E)=\frac{1}{2}+\frac{1}{2} \cos 2 \theta_{\text {Sun }} \cos 2 \theta_{0}, \\
P_{\text {night }}\left(E ; \Theta_{\mathrm{N}}\right)=\frac{1}{2}+\frac{1}{2} \cos 2 \theta_{\text {Sun }}\left\{\cos 2 \theta_{n}^{-}+2 \sin 2 \theta_{0} \sum_{j=1}^{n-1} \Delta \theta_{j} \cos 2 \Delta \psi_{n, j}\right\} .
\end{gathered}
$$

The notation in the above expressions is as follows. At night, for a given nadir angle $\Theta_{\mathrm{N}}$, the neutrino crosses $n=n\left(\Theta_{\mathrm{N}}\right)$ interfaces, including the air-to-crust $(j=1)$ and the crust-to-air $(j=n)$ interfaces, where the potential is discontinuous and the effective mixing angle in the medium experiences jumps $\Delta \theta_{j} ; \Delta \psi_{n, j}=\Delta \psi_{n, j}\left(\Theta_{N} ; E\right)$ is the oscillation phase incursion from the $j$ th to the $n$th crossing point, i.e., to the detector [8]; $\theta_{n}^{-}$and $\theta_{\text {Sun }}$ are the effective mixing angles in the crust under the detector and in the solar core, respectively.

The oscillation phases in the nighttime observation probability rapidly change with time, thus, $P_{\text {night }}$ contains one cumulative (constant) and a number of rapidly oscillating contributions. When integrated over time (possibly with a smooth enough weight function $w(t)$ ), the oscillatory ones are smeared out, but not quite completely, as estimated by the well-known stationary phase approximation

$$
\int_{a}^{b} F(t) e^{\mathrm{i} \lambda S(t)} \mathrm{d} t \approx \sqrt{\frac{2 \pi \mathrm{i}}{\lambda S^{\prime \prime}\left(t_{0}\right)}} F\left(t_{0}\right) e^{\mathrm{i} \lambda S\left(t_{0}\right)}+\left.\frac{F(t) e^{\mathrm{i} \lambda S(t)}}{\mathrm{i} \lambda S^{\prime}(t)}\right|_{a} ^{b}, \quad \lambda \rightarrow \infty
$$

(assuming that there is only one stationary point $t_{0} \in[a, b]$ such that $\left.S^{\prime}\left(t_{0}\right)=0\right)$. The above approximation is indeed applicable to solar neutrinos inside the Earth, since the oscillation phases $2 \Delta \psi_{n, j} \propto 2 \lambda r_{j} \gg 2 \pi\left(r_{j}\right.$ is the radius of the $j$ th interface); this is the reason for using the same notation $\lambda$ for a large parameter in (4). Moreover, the boundary term vanishes in (4), since the integration endpoints for the contribution of the $j$ th interface in Eq. (3) correspond to a neutrino passing tangentially to it, where $S^{\prime}(t) \rightarrow \infty$. The leading contribution to the net day-night asymmetry from the oscillatory term in (3) comes from the stationary points, which are the midnights during the night and the two solstices during the year [9].

Although the contributions of the stationary points are suppressed as $\lambda^{-1 / 2}$, they have an unusual property that lets one extract them from under the Poisson noise. Namely, these contributions come from small neighborhoods of the stationary points (i.e., they are localized) and the corresponding localization scale $\delta t$ is such that $\left|S\left(t_{0}+\delta t\right)-S\left(t_{0}\right)\right| \sim 2 \pi / \lambda$; for ${ }^{8} \mathrm{~B}$ neutrinos, typical localization scales are about a couple of hours around the midnights 
and 1-2 months around the two solstices. Due to localization of these contributions, if one shrinks the observation time window $\Delta t$ down to $\delta t$, then the contribution of the stationary point to the time integral will almost remain unchanged, while its contribution to the time average will increase proportionally to $1 / \Delta t$. Note that nothing like occurs to the cumulative contribution. Moreover, relative statistical uncertainties grow as $1 / \sqrt{\Delta t}$, so, somewhat counterintuitively, the signal-to-noise ratio for the stationary-point contributions is improved for reduced observation time windows (for details, incl. the localization scales, see Ref. [8]).

\section{Numerical simulation of the temporally-weighted day-night effect}

Based on the idea described above, we made a numerical estimation of the day-night asymmetry to be observed at a typical water Cherenkov detector with temporal weighting of events introduced to smoothly select those around the winter solstice stationary point (the summer solstice gives a smaller contribution [8]). One should start here with the definitions of the temporally-weighted quantities. Namely, from the weighted numbers of nighttime/daytime neutrino events $N_{\text {night,day }}^{(w)}=\sum_{k} \vartheta\left( \pm \pi / 2 \mp \Theta_{\mathrm{N}}\left(t_{k}\right)\right) w\left(t_{k}\right)$ observed at times $t_{k}$, one can construct the 'experimental' asymmetry factor

$$
\mathcal{A}_{\text {dn }}^{(w)}=\frac{2\left(N_{\text {night }}^{(w)} / \Delta t_{\text {night }}-\dot{\bar{N}}_{\text {day }}\right)}{N_{\text {night }}^{(w)} / \Delta t_{\text {night }}+\dot{\bar{N}}_{\text {day }}},
$$

where $\Delta t_{\text {night }}=\int \vartheta\left(\pi / 2-\Theta_{\mathrm{N}}(t)\right) w(t) \mathrm{d} t$ is the weighted total observation time at night and $\dot{\bar{N}}_{\text {day }}$ is the average daytime rate of neutrino events, which is constant and does not depend on weighting. Per narrow recoil energy bin $[T, T+\Delta T], \Delta T \rightarrow 0$, the above asymmetry factor can be expressed in terms of the 'probabilistic' asymmetry independent of the detector [8],

$$
\begin{aligned}
\mathcal{A}_{\mathrm{dn}}^{(w)} \approx A_{\mathrm{dn}, \text { mono }}^{(w)}(T) & =\frac{\int \Phi(E) \mathrm{d} E \Delta \frac{\mathrm{d} \sigma(E, T)}{\mathrm{d} T} P_{\mathrm{day}}(E) \hat{A}_{\mathrm{dn}}^{(w)}(E)}{\int \Phi(E) \mathrm{d} E\left\{\Delta \frac{\mathrm{d} \sigma(E, T)}{\mathrm{d} T} P_{\mathrm{day}}(E)+\frac{\mathrm{d} \sigma_{v_{x}}(E, T)}{\mathrm{d} T}\right\}}, \\
\hat{A}_{\mathrm{dn}}^{(w)}(E) & =\frac{2\left[\left\langle P_{\text {night }}(E)\right\rangle_{w}-P_{\text {day }}(E)\right]}{\left\langle P_{\text {night }}(E)\right\rangle_{w}+P_{\text {day }}(E)},
\end{aligned}
$$

where $\Phi(E)$ is the solar neutrino energy spectrum, while $\mathrm{d} \sigma_{v_{x}}(E, T)$ and $\mathrm{d} \sigma_{v_{e}}(E, T) \equiv$ $\mathrm{d} \sigma_{v_{e}}(E, T)+\Delta \mathrm{d} \sigma(E, T)$ are the detection cross sections for neutrinos in the two flavor states.

Even though neutrino energies $E$ and the 'probabilistic' asymmetry factor are unobservable in elastic scattering channels, such as those used in SuperK and Borexino detectors, this factor lets one clearly see how contibutions of the stationary points get enhanced for reduced observation windows (see Fig. 1). The effect for nearly-monochromatic ${ }^{7}$ Be neutrinos shown in the inset has been recently studied in Ref. [10]. It is vivid that the amplitude of the localized contribution becomes even greater than the cumulative contribution if one shrinks the observation sessions to hours around midnights during the two winter months around the solstice. It is also worth mentioning that, unlike the cumulative effect, the localized one depends quite strongly on the latitude of the detector, experiencing a substantial amplification around the tropical latitude $\chi \approx 23.4^{\circ}[8,9]$.

One could clearly argue that all the oscillatory signatures one can see in Fig. 1 will be integrated out when one resorts to energy distributions of recoil electrons that are actually observed in experiments. In order to estimate the degree of validity of this claim, we have calculated the 'experimental' day-night asymmetry factor (6) as a function of the recoil en$\operatorname{ergy} T$, for a tropical detector $\chi=23.4^{\circ}$ and a detector at the latitude of SuperK $\chi=36.2^{\circ}$ 


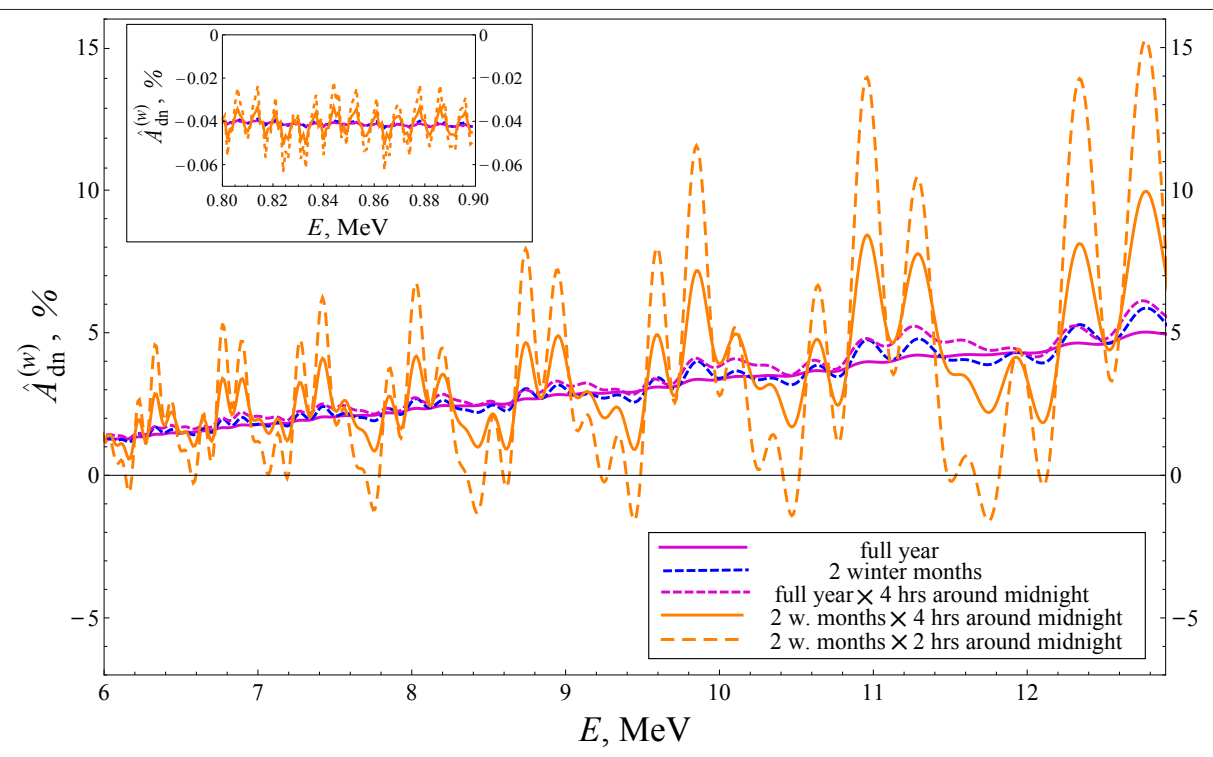

Figure 1. The 'probabilistic' asymmetry (7) versus the neutrino energy $E$ depending on the observation time window for a detector at the latitude $\chi=26^{\circ}$. Inset: the same for the energies of ${ }^{7} \mathrm{Be}$ neutrinos (around $E=0.862 \mathrm{MeV}$ )

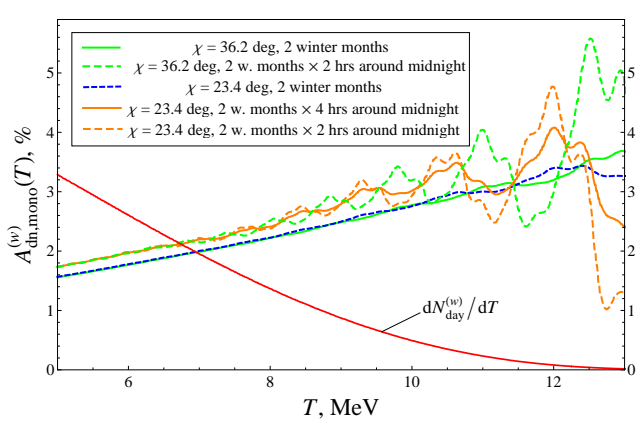

(a)

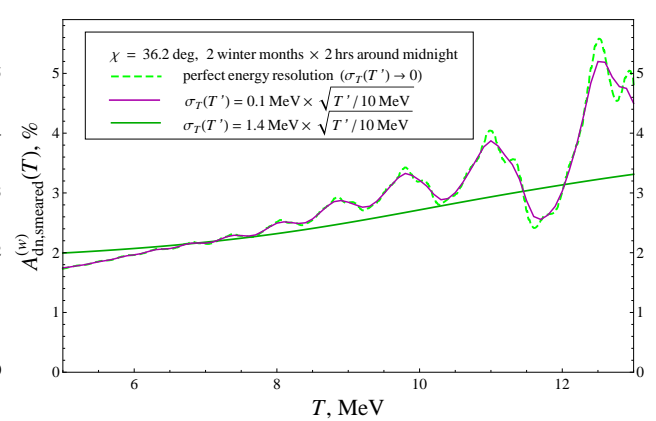

(b)

Figure 2. (a) The 'experimental' asymmetry (6) versus the electron recoil energy $T$ depending on the observation time window and the latitude $\chi$ of the detector. The solid red curve shows the recoil energy distribution of the neutrino events in arbitrary units. (b) The same but smeared over a finite recoil energy resolution of the detector

(Fig. 2(a)). From this figure, one observes that the high-energy tail of the recoil energy spectrum is affected and the signature of the effect depends significantly on the latitude. Of course, this signature is hard to observe because only few recoil electrons will have such high energies (it would take about 12 years for a Super-Kamiokande event rate to see the effect above the statistical noise [8]). Moreover, the recoil energy resolution of Super-Kamiokande is not enough to resolve the 'wiggles' in Fig. 2(a). However, a resolution of a JUNO-class detector [11] would be definitely enough for that (see Fig. 3(b)). Observation of such an interferencelike effect, namely, of a peak or a dip in the high-energy segment of the weighted day-night 
asymmetry, could also serve as a precision test of the neutrino mass-squared difference, as clearly seen from Fig. 3.

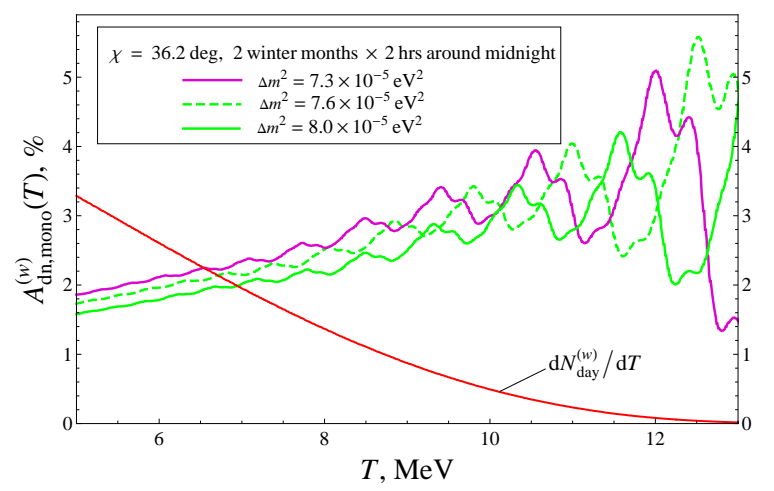

Figure 3. Signatures on the weighted 'experimental' asymmetry (6) for different neutrino mass-squared differences $\Delta m^{2}$

Instead of a conclusion, we would like to make it clear that the effect we have described above does not have to be visible at the Super-Kamiokande detector because of a higher energy resolution and a larger number of events it requires. However, we cannot help noting quite an interesting high-energy peak and a visually 'wiggly' pattern in the recoil energy distribution observed at SuperK (see, e.g., Fig. 1 in Ref. [12]). These signatures are not quite statistically significant, but what will take place if one applies temporal weighting to extract them from under the noise?

\section{Acknowledgments}

The numerical simulations reported have been performed using the Supercomputing Cluster "Lomonosov" of Moscow State University [13].

\section{References}

[1] E. D. Carlson, Phys. Rev. D 34, 1454 (1986).

[2] A. J. Baltz and J. Weneser, Phys. Rev. D 35, 528 (1987).

[3] A. Renshaw et al., Phys. Rev. Lett. 112, 091805 (2014).

[4] A. M. Dziewonski and D. L. Anderson, Phys. Earth Planet. Inter. 25, 297 (1981).

[5] L. Wolfenstein, Phys. Rev. D 17, 2369 (1978).

[6] A. D. Supanitsky, J. C. D’Olivo, and G. A. Medina-Tanco, Phys. Rev. D 78, 045024 (2008).

[7] P. C. de Holanda, Wei Liao, and A. Yu. Smirnov, Nucl. Phys. B 702, 307 (2004).

[8] O. G. Kharlanov, e-Print arXiv:1509.08073[hep-ph].

[9] S. S. Aleshin, O. G. Kharlanov, and A. E. Lobanov, Phys. Rev. D 87, 045025 (2013).

[10] A. N. Ioannisian, A. Yu. Smirnov, and D. Wyler, Phys. Rev. D 92, 013014 (2015).

[11] Y. F. Li, Int. J. Mod. Phys. Conf. Ser. 31, 1460300 (2014).

[12] M. B. Smy for Super-Kamiokande Collaboration, Nucl. Part. Phys. Proceedings 265, 135 (2015).

[13] V. Sadovnichy et al., in Contemporary High Performance Computing: From Petascale toward Exascale (CRC Press, Boca Raton, USA, 2013), p. 283. 